\title{
Protocol-Aware Design Criteria and Performance Analysis for Distributed Space-Time Coding
}

\author{
Karim G. Seddik, Ahmed K. Sadek, and K. J. Ray Liu \\ Department of Electrical and Computer Engineering, \\ and Institute for Systems Research \\ University of Maryland, College Park, MD 20742, USA. \\ \{kseddik, aksadek, kjrliu\}@eng.umd.edu
}

\begin{abstract}
In this paper, we consider the design of distributed space-time codes for wireless networks. Distributed spacetime coding (DSTC) can be achieved through node cooperation to emulate multiple transmit antennas. We derive the distributed space-time codes design criteria for different scenarios based on the pairwise error probability (PEP) analysis. First, we consider the decode-and-forward (DAF) protocol and prove that space-time codes, designed to achieve full diversity and maximum coding gain in the MIMO channels, will achieve full diversity but not necessarily maximizing the coding gain if used with the DAF protocol. Next, we consider the amplifyand-forward (AAF) protocol and prove that a space-time code designed to achieve full diversity and maximum coding gain in MIMO channels will achieve the same if used with the AAF protocol.
\end{abstract}

\section{INTRODUCTION}

Designing protocols that allow several single-antenna terminals to cooperate via forwarding each others' data can increase the system reliability through achieving spatial diversity. In [1] and [2], various cooperative protocols were proposed and outage probability analysis for these protocols was provided. The concepts of DAF and AAF have been introduced in these works. The problem with these protocols is the loss in the data rate as the number of relays increases and this leads to the use of what is known as distributed space-time coding. The term distributed comes from the fact that the virtual multi-antenna transmitter is distributed between randomly placed relay nodes. It was proposed in [1] to use relay nodes to form a virtual multiantenna transmitter to achieve diversity and outage analysis was presented for the system. Previous work [3]-[5] have considered the use of the existing space-time codes used for the MIMO channels to be deployed in a distributed manner. These works did not take into consideration the code design criteria for the space-time codes when they are employed in a distributed fashion. In this paper, we consider the design of the distributed space-time codes, with the DAF (or AFF) protocol, by deriving the code design criteria to achieve full diversity and to maximize the coding gain. We compare the derived code design criteria with those for the space-time codes used over MIMO channels. We try to answer the question whether the space-time code which achieves full diversity and maximum coding gain in the MIMO channels can also achieve full diversity and maximum coding gain if it is used in a distributed fashion.

\section{DSTC WITH THE DAF PROTOCOL}

The system has two phases, in phase 1 , if $n$ relays are assigned for cooperation, the source transmits data to these relays with power $P_{1}$ and the signal received at the $i$-th relay can be modeled as

$$
\mathbf{y}_{s, r_{i}}=\sqrt{P_{1}} h_{s, r_{i}} \mathbf{s}+\mathbf{v}_{s, r_{i}}, \quad i=1,2, \cdots, n,
$$

where $\mathbf{s}$ is an $L_{n} \times 1\left(L_{n} \geq n\right)$ transmitted data vector with average energy $E\left[\|\mathbf{s}\|^{2}\right] \leq L_{n}$, and $h_{s, r_{i}}$ denotes the channel gain between the source and the $i$-th relay and it follows a circularly symmetric complex Gaussian random variable with zero mean and variance $\delta_{s, r}^{2}$ which we denote by $\mathcal{C N}\left(0, \delta_{s, r}^{2}\right)$. The channel gains from the source to the relays are assumed i.i.d. All channels are fixed during the transmission of one data packet and can vary from one packet to another. In (1), $\mathbf{v}_{s, r_{i}} \sim \mathcal{C N}\left(0, N_{o}\right)$ denotes additive white Gaussian noise (AWGN). The $n$ relays try to decode the received signals. We assume that the relay will be able to decide whether it decoded correctly or not. If a relay decodes correctly it will forward the data in the second phase of the cooperation protocol, otherwise it remains idle. The relays are assumed to be synchronized either by a centralized or a distributed algorithm.

In phase 2 , the relays that decodes correctly re-encodes the data vector $\mathbf{s}$ with a pre-assigned code structure. The ST code is distributed among the relays such that each relay will emulate a single antenna in a multiple antenna transmitter. Let $\mathbf{x}_{r_{i}}$ denote the $K_{n} \times 1$ code generated by the $i$-th relay with $K_{n} \geq L_{n}$. Hence the signal received at the destination from all relays can be modeled as

$$
\mathbf{y}_{\mathbf{d}}=\sqrt{P_{2}}\left[I_{1} \mathbf{x}_{r_{1}}, I_{2} \mathbf{x}_{r_{2}}, \cdots, I_{n} \mathbf{x}_{r_{n}}\right] \mathbf{h}_{d}+\mathbf{v}_{d}
$$

where $\mathbf{h}_{d}=\left[h_{r_{1}, d} h_{r_{2}, d} \cdots h_{r_{n}, d}\right]^{T}$ is an $n \times 1$ vector channel gains from the $n$ relays to the destination and $h_{r_{i}, d} \sim \mathcal{C N}\left(0, \delta_{r, d}^{2}\right)$ and $P_{2}$ is the relay power. The $n$ 
channels are assumed to be statistically independent as the relays are spatially separated, and $\mathbf{v}_{d}$ denotes AWGN at the destination and has i.i.d. entries with zero mean and variance $N_{o}$. The state of the $k$-th relay, i.e., whether it decoded correctly or not, is denoted by the random variable $I_{k}(1 \leq k \leq n)$ which takes values 1 or 0 if the relay decodes correctly or erroneously, respectively. The random variables $I_{k}$ 's $(1 \leq k \leq n)$ are statistically independent as the state of each relay depends only on its channel conditions to the source which are independent from other relays. There is an energy constraint on the generated ST code such as $E\left[\left\|\mathbf{X}_{\mathbf{r}}\right\|^{2}\right] \leq K_{n}$, where $\mathbf{X}_{\mathbf{r}}$ is an $K_{n} \times n$ ST code matrix with the $k$-th column being the ST code vector $\mathbf{x}_{r_{k}}$ transmitted by the $k$-th relay if it decoded correctly.

The received signal model at the destination in (2) can be rewritten as follows

$$
\mathbf{y}_{\mathbf{d}}=\sqrt{P_{2}} \mathbf{X}_{\mathbf{r}} \mathbf{h}_{d, \mathbf{I}}+\mathbf{v}_{d}
$$

The new channel definition $\mathbf{h}_{d, \mathbf{I}}$ includes the information about both the channel realization from the $n$ relays to the destination along with the relay state information $\mathbf{I}=$ $\left[I_{1}, I_{2}, \cdots, I_{n}\right]$ and is defined as follows

$$
\mathbf{h}_{d, \mathbf{I}}=\left[I_{1} h_{r_{1}, d}, I_{2} h_{r_{2}, d}, \cdots, I_{n} h_{r_{n}, d}\right]^{T} .
$$

Hence, if the $k$-th relay decoded in error then its equivalent channel $I_{k} h_{r_{k}, d}$ equals 0 .

The random variable $I_{k}$ is a Bernoulli random variable with a distribution given by

$$
I_{k}= \begin{cases}0 & \text { with probability } \simeq L_{n} \mathbf{S E R} \\ 1 & \text { with probability } \simeq 1-L_{n} \mathbf{S E R},\end{cases}
$$

where the probability of decoding a packet of length $L_{n}$ in error is approximated using the union-bound by $L_{n} \mathbf{S E R}$, where SER is the symbol error rate and is modulation dependent. For $M$-ary quadrature amplitude modulation ( $M$-QAM, $M=2^{k}$ with $k$ even), we can show that the exact expression can be upper bounded by

$$
\mathbf{S E R} \leq \frac{N_{o} g(2)}{b P_{1} \delta_{s, r}^{2}}
$$

where $b=3 /(M-1)$ and $g(2)=\frac{4 K}{\pi} \int_{0}^{\pi / 2} \sin ^{2} \theta d \theta-$ $\frac{4 K^{2}}{\pi} \int_{0}^{\pi / 4} \sin ^{2} \theta d \theta$ in which $K=1-\frac{1}{\sqrt{M}}$. The proof is omitted due to space limitations.

The destination has full channel state information $\mathbf{h}_{d, \mathbf{I}}$ and applies a maximum likelihood (ML) receiver. We can show that the conditional PEP given the equivalent channel realization $\mathbf{h}_{d, \mathbf{I}}$ and the number of relays $n$ can be upper bounded as follows

$$
P\left(\mathbf{X} \rightarrow \hat{\mathbf{X}} \mid \mathbf{h}_{d, I}\right) \leq \exp \left(-\frac{P_{2}\left\|\mathbf{\Phi}(\mathbf{X}, \hat{\mathbf{X}}) \mathbf{h}_{d, I}\right\|^{2}}{4 N_{o}}\right)
$$

where $\mathbf{\Phi}(\mathbf{X}, \hat{\mathbf{X}})=(\mathbf{X}-\hat{\mathbf{X}})$ is the difference matrix between the two codewords $\mathbf{X}$ and $\hat{\mathbf{X}}$ and $\|\cdot\|^{2}$ is the Frobenius norm of a matrix. The Frobenius norm in (7) can be further decomposed as

$$
\left\|\mathbf{\Phi}(\mathbf{X}, \hat{\mathbf{X}}) \mathbf{h}_{d, I}\right\|^{2}=\mathbf{h}_{d}^{\mathcal{H}} \mathbf{D}_{\mathbf{I}} \mathbf{\Phi}(\mathbf{X}, \hat{\mathbf{X}})^{\mathcal{H}} \boldsymbol{\Phi}(\mathbf{X}, \hat{\mathbf{X}}) \mathbf{D}_{\mathbf{I}} \mathbf{h}_{d},
$$

where $\mathbf{D}_{\mathbf{I}}$ is an $n \times n$ diagonal matrix with the $k$-th diagonal entry equal to $I_{k}$. Let $r_{\mathbf{I}}$ denote the rank of the matrix $\boldsymbol{\Phi}(\mathbf{X}, \hat{\mathbf{X}}) \mathbf{D}_{\mathbf{I}}$, which is the difference code matrix after inserting zeros in the columns corresponding to the relays that decoded in error. This rank is generally less than or equal to the number of relays that decode correctly, which we denote by $c_{\mathbf{I}}$. The matrix $\mathbf{D}_{\mathbf{I}} \mathbf{\Phi}(\mathbf{X}, \hat{\mathbf{X}})^{\mathcal{H}} \boldsymbol{\Phi}(\mathbf{X}, \hat{\mathbf{X}}) \mathbf{D}_{\mathbf{I}}$ is hermitian and can thus be decomposed into

$$
\mathbf{D}_{\mathbf{I}} \boldsymbol{\Phi}(\mathbf{X}, \hat{\mathbf{X}})^{\mathcal{H}} \mathbf{\Phi}(\mathbf{X}, \hat{\mathbf{X}}) \mathbf{D}_{\mathbf{I}}=\mathbf{U} \boldsymbol{\Lambda} \mathbf{U}^{\mathcal{H}}
$$

where $\mathbf{U}$ is a unitary matrix with the $i$-th column $\mathbf{u}_{i}$ denoting the $i$-th eigenvector and $\boldsymbol{\Lambda}$ is a diagonal matrix with the $i$-th entry $\lambda_{i}$ denoting the $i$-th eigenvalue which are arranged in a non-increasing order. We can write (8) using the decomposition in (9) as follows

$$
\mathbf{h}_{d}^{\mathcal{H}} \mathbf{U} \boldsymbol{\Lambda} \mathbf{U}^{\mathcal{H}} \mathbf{h}_{d}=\tilde{\mathbf{h}}_{d}^{\mathcal{H}} \boldsymbol{\Lambda} \tilde{\mathbf{h}}_{d},
$$

where $\tilde{\mathbf{h}}_{d}=\mathbf{U}^{\mathcal{H}} \mathbf{h}_{d}$. Since $h_{r_{i}, d}$ 's are independent and identically distributed Gaussian random variables, and the matrix $\mathbf{U}$ is unitary, then the elements of the vector $\tilde{\mathbf{h}}_{d}$ are also Gaussian and independent. The random vectors $\tilde{\mathbf{h}}_{d}$ and $\mathbf{I}$ are mutually independent as they arise from independent processes. First, we average over the transformed channel realizations $\tilde{\mathbf{h}}_{d}$. The channel gain $\left|\tilde{h}_{d}(i)\right|^{2}$, has an exponential distribution with parameter $1 / \delta_{r, d}^{2}$. Averaging the conditional PEP in (7) over all channel realizations, we get

$$
P(\mathbf{X} \rightarrow \hat{\mathbf{X}} \mid \mathbf{I}) \leq \prod_{i=1}^{r_{\mathbf{I}}} \frac{1}{1+\frac{P_{2} \delta_{r, d}^{2}}{4 N_{o}} \lambda_{\mathbf{I}_{i}}},
$$

where $\left\{\lambda_{\mathbf{I}_{i}}\right\}_{i=1}^{r_{\mathbf{I}}}$ is a subset of the eigenvalues of the matrix $\mathbf{D}_{\mathbf{I}} \boldsymbol{\Phi}(\mathbf{X}, \hat{\mathbf{X}})^{\mathcal{H}} \boldsymbol{\Phi}(\mathbf{X}, \hat{\mathbf{X}}) \mathbf{D}_{\mathbf{I}}$ that depends on the realization of $\mathbf{I}$.

Second, we average over different realizations of the relays states $\mathbf{I}$. The dependence of the expression in (11) on $\mathbf{I}$ appears in the upper limit of the summation $r_{\mathbf{I}}$ and the set of non-zero eigenvalues $\left\{\lambda_{\mathbf{I}_{i}}\right\}_{i=1}^{r_{\mathbf{I}}}$. To take into account the dependence of the set of non-zero eigenvalues $\left\{\lambda_{\mathbf{I}_{i}}\right\}_{i=1}^{r_{I}}$ on the realization of the relays states $\mathbf{I}$, i.e., which relays decoded correctly, we upper bound the PEP in (11) as follows

$$
P\left(\mathbf{X} \rightarrow \hat{\mathbf{X}} \mid \mathbf{I}, c_{\mathbf{I}}=k\right) \leq \max _{\mathcal{I}_{k}: c_{\mathbf{I}}=k} \prod_{i=1}^{r_{\mathbf{I}}} \frac{1}{1+\frac{P_{2} \delta_{r, d}^{2}}{4 N_{o}} \lambda_{\mathbf{I}_{i}}},
$$


where $\mathcal{I}_{k}$ denotes the set of realizations $\mathbf{I}$ which have the same number of relays that decoded correctly $k$. If we consider the high SNR performance, the set of eigenvalues that maximizes the PEP bound in (12) will clearly correspond to the set of eigenvalues of the matrix $\mathbf{D}_{\mathbf{I}} \boldsymbol{\Phi}(\mathbf{X}, \hat{\mathbf{X}})^{\mathcal{H}} \boldsymbol{\Phi}(\mathbf{X}, \hat{\mathbf{X}}) \mathbf{D}_{\mathbf{I}}$ with the smallest rank. We denote these eigenvalues by $\left\{\lambda_{i, k}\right\}_{i=1}^{r_{k}}$ and generally $r_{k} \leq k$.

Since the $I_{k}$ 's are i.i.d. Bernoulli r.v.'s as in (5), the number of relays that decoded correctly $c_{\mathbf{I}}$ has a binomial distribution given by

$$
P_{c_{\mathbf{I}}}(k)=\left(\begin{array}{l}
n \\
k
\end{array}\right)\left(1-L_{n} \mathbf{S E R}\right)^{k}\left(L_{n} \mathbf{S E R}\right)^{n-k} .
$$

Define the SNR as $S N R=P / N_{o}$, where $P=P_{1}+P_{2}$ is the total power used in transmission from the source and the relays. Let the ratios of the total power assigned to the source and the relays be denoted by $a_{1}$ and $a_{2}$, respectively, where $P_{1}=a_{1} P$ and $P_{2}=a_{2} P$ where $\alpha_{1}+\alpha_{2}=1$. Averaging over all realizations of the states of the relays and considering high SNR we get

$$
\begin{gathered}
P(\mathbf{X} \rightarrow \hat{\mathbf{X}}) \leq \sum_{k=0}^{n}\left(\begin{array}{l}
n \\
k
\end{array}\right) S N R^{-n+\left(k-r_{k}\right)}\left(\frac{L_{n} g(2)}{b a_{1} \delta_{s, r}^{2}}\right)^{n-k} \\
\cdot \prod_{i=1}^{r_{k}}\left(\frac{a_{2} \delta_{r, d}^{2}}{4} \lambda_{i, k}\right)^{-1}
\end{gathered}
$$

The diversity gain achieved is defined as $d=\lim _{S N R \rightarrow \infty}-$ $\frac{\log (P E P(S N R))}{\log (S N R)}$. Applying this definition to the conditional PEP in (14), when the number of cooperating nodes is $n$, we get

$d_{D F}=\lim _{S N R \rightarrow \infty}-\frac{\log (P E P)}{\log (S N R)}=\min _{k \in\{1, \cdots, n\}} n-\left(k-r_{k}\right)$.

From (15) it is clear that the maximum achievable diversity is $n$ and is achieved when $r_{k}=k \forall k$. This maximum diversity can only be achieved if the matrix $\mathbf{\Phi}(\mathbf{X}, \hat{\mathbf{X}})^{\mathcal{H}} \boldsymbol{\Phi}(\mathbf{X}, \hat{\mathbf{X}})$ has a full rank of order $\mathrm{n}$. Then the matrix $\mathbf{D}_{\mathbf{I}} \mathbf{\Phi}(\mathbf{X}, \hat{\mathbf{X}})^{\mathcal{H}} \boldsymbol{\Phi}(\mathbf{X}, \hat{\mathbf{X}}) \mathbf{D}_{\mathbf{I}}$ has always a rank of $c_{\mathbf{I}}$, which is the number of relays that decode correctly, independent of the specific realization of $\mathbf{I}$ with the same $c_{\mathbf{I}}$. If the full diversity is achieved, the coding gain is

$C_{D F}=\left(\sum_{k=0}^{n}\left(\begin{array}{l}n \\ k\end{array}\right)\left(\frac{L_{n} g(2)}{b a_{1} \delta_{s, r}^{2}}\right)^{n-k} \prod_{i=1}^{k}\left(\frac{4}{a_{2} \delta_{r, d}^{2} \lambda_{i, k}}\right)\right)^{-\frac{1}{n}}$,

which is a term that does not depend on the SNR. To minimize PEP bound we need to maximize the coding gain of the distributed space-time code. So to achieve full diversity of order $n$ the code matrix $\mathbf{\Phi}(\mathbf{X}, \hat{\mathbf{X}})^{\mathcal{H}} \mathbf{\Phi}(\mathbf{X}, \hat{\mathbf{X}})$ must be of full column rank over all pairs of distinct codewords $\mathbf{X}$ and
$\hat{\mathbf{X}}$. This is the same code design criterion as for the spacetime codes designed for the MIMO channels to achieve full diversity [6].

To maximize the coding gain of the distributed spacetime code we need for each $k \in\{1, \cdots, n\}$ to maximize $\min _{\aleph: \aleph \subset\{1, \cdots, n\},|\aleph|=k}\left(\prod_{i=1}^{k} \lambda_{i, \aleph}\right)$, where $|\aleph|$ indicates the cardinality of the subset $\aleph$ and $\lambda_{i, \aleph}$ is the $i$-th eigenvalue of the code matrix corresponding to the subset $\aleph$ of relays that have decoded correctly. The maximization is over all distinct pairs of codewords $\mathbf{X}$ and $\hat{\mathbf{X}}$. This is different from the determinant criterion in the case of MIMO channels [6]. Intuitively, the difference is due to the fact that in the case of distributed space-time codes, not all the relays will always transmit their corresponding code matrix columns. The design criterion used in the case of distributed spacetime codes makes sure that the coding gain is significant over all sets of possible relays that have decoded correctly in the first phase.

\section{DSTC WITH THE AAF PROTOCOL}

The system has two phases, in phase 1 , if $n$ relays are assigned for cooperation, the source transmits data to these relays with power $P_{1}$ and the signal received at the $i$-th relay can be modeled as

$$
\mathbf{y}_{s, r_{i}}=\sqrt{P_{1}} h_{s, r_{i}} \mathbf{s}+\mathbf{v}_{s, r_{i}}, \quad i=1,2, \cdots, n,
$$

where $\mathbf{s}$ is a $n \times 1$ transmitted data vector with average energy $E\left[\|\mathbf{s}\|^{2}\right] \leq n$. the channel and the noise are modeled as in (1). In the AAF protocol, the relays do not decode the received signal. Instead, the relays can only amplify the received signal and perform simple operations such as permutations of the received symbols or other forms of linear transformations. We constraint the linear transformations to have rows of unit norm which means that the average power transmitted by a single relay is the same for any time slot. Let $\tilde{\mathbf{X}}$ denote the $K_{n} \times n$ code generated by the $n$ relays if the system was noise free with $K_{n} \geq n$. Each relay will multiply the received signal by the factor $\sqrt{\frac{P_{2} / K_{n}}{P_{1} \delta_{s, r}^{2}+N_{0}}}$. It can be easily shown that this normalization will give a transmitted power per symbol $P=P_{1}+P_{2}$ [5]. Hence, the signal received at the destination from all relays can be modeled as

$$
\mathbf{y}_{\mathbf{d}}=\sqrt{\frac{P_{2} / K_{n}}{P_{1} \delta_{s, r}^{2}+N_{0}}} \tilde{\mathbf{X}} \mathbf{h}_{d}+\mathbf{v}_{d}
$$

where $\mathbf{h}_{d}=\left[h_{r_{1}, d}, h_{r_{2}, d}, \cdots, h_{r_{n}, d}\right]^{T}$ is an $n \times 1$ vector channel gains from the $n$ relays to the destination and $h_{r_{i}, d} \sim \mathcal{C N}\left(0, \delta_{r, d}^{2}\right)$. $\mathbf{v}_{d}$ denotes additive white Gaussian noise AWGN. Each element of $\mathbf{v}_{d}$ is $\mathcal{C N}\left(0, N_{0}\left(1+\frac{P_{2} / K_{n}}{P_{1} \delta_{s, r}^{2}+N_{o}} \sum_{i=1}^{n}\left|h_{r_{i}, d}\right|^{2}\right)\right)$, and $\mathbf{v}_{d}$ accounts for both the noise propagated from the relay nodes as 
well as the noise generated at the destination. The received vector in (18) can be written as

$$
\mathbf{y}_{\mathbf{d}}=\sqrt{\frac{P_{2} P_{1} / K_{n}}{P_{1} \delta_{s, r}^{2}+N_{0}}} \mathbf{X H}+\mathbf{v}_{d},
$$

where $\mathbf{H}=\left[h_{s, r_{1}} h_{r_{1}, d}, h_{s, r_{2}} h_{r_{2}, d}, \cdots, h_{s, r_{n}} h_{r_{n}, d}\right]^{T}$. Now $\mathbf{X}$ plays the role of the space-time codeword.

With the ML decoder, the PEP of mistaking $\mathbf{X}$ by $\hat{\mathbf{X}}$ can be upper bounded by the following Chernoff bound

$$
\begin{aligned}
& P(\mathbf{X} \rightarrow \hat{\mathbf{X}}) \leq \\
& E_{\mathbf{H}} e^{-\frac{P_{1} P_{2} / K_{n}}{4 N_{0}\left(P_{1} \delta_{s, r}^{2}+N_{o}+\frac{P_{2}}{K_{n}} \sum_{i=1}^{n}\left|h_{r_{i}, d}\right|^{2}\right)}} \mathbf{H}^{\mathcal{H}}(\mathbf{X}-\hat{\mathbf{X}})^{\mathcal{H}}(\mathbf{X}-\hat{\mathbf{X}}) \mathbf{H}
\end{aligned}
$$

By averaging over the source to relay channels we get [5]

$$
\begin{aligned}
& P(\mathbf{X} \rightarrow \hat{\mathbf{X}}) \leq E_{h_{r_{1}, d}, \cdots, h_{r_{n}, d}} \operatorname{det}^{-1}[ \\
& \mathbf{I}_{n}+\frac{\delta_{s, r}^{2} P_{1} P_{2} / K_{n}}{4 N_{0}\left(P_{1} \delta_{s, r}^{2}+N_{o}+\frac{P_{2}}{K_{n}} \sum_{i=1}^{n}\left|h_{r_{i}, d}\right|^{2}\right)} \\
& \left.\quad(\mathbf{X}-\hat{\mathbf{X}})^{\mathcal{H}}(\mathbf{X}-\hat{\mathbf{X}}) \operatorname{diag}\left(\left|h_{r_{1}, d}\right|^{2},\left|h_{r_{2}, d}\right|^{2}, \cdots,\left|h_{r_{n}, d}\right|^{2}\right)\right],
\end{aligned}
$$

where $\mathbf{I}_{n}$ is the $n \times n$ identity matrix.

Define the matrix

$$
\begin{aligned}
\mathbf{M}= & \frac{\delta_{s, r}^{2} P_{1} P_{2} / K_{n}}{4 N_{0}\left(P_{1} \delta_{s, r}^{2}+N_{o}+\frac{P_{2}}{K_{n}} \sum_{i=1}^{n}\left|h_{r_{i}, d}\right|^{2}\right)} \\
& \Psi(\mathbf{X}, \hat{\mathbf{X}}) \operatorname{diag}\left(\left|h_{r_{1}, d}\right|^{2},\left|h_{r_{2}, d}\right|^{2}, \cdots,\left|h_{r_{n}, d}\right|^{2}\right),
\end{aligned}
$$

where $\Psi(\mathbf{X}, \hat{\mathbf{X}})=(\mathbf{X}-\hat{\mathbf{X}})^{\mathcal{H}}(\mathbf{X}-\hat{\mathbf{X}})$. The bound in (21) can be written in terms of the eigenvalues of $\mathbf{M}$ as

$$
P(\mathbf{X} \rightarrow \hat{\mathbf{X}}) \leq E_{h_{r_{1}, d}, \cdots, h_{r_{n}, d}} \frac{1}{\prod_{i=1}^{n}\left(1+\lambda_{M_{i}}\right)},
$$

where $\lambda_{M_{i}}$ is the $i$-th eigenvalue of the matrix $\mathbf{M}$. If we let $P_{1}=\alpha P$ and $P_{2}=(1-\alpha) P$ where $P$ is the power per symbol for some $\alpha \in(0,1)$ and define $S N R=P / N_{0}$, we can easily see that the eigenvalues of $\mathbf{M}$ increase with the increase of the SNR. We will now assume that the matrix $\mathbf{M}$ has full rank of order $n$. At high SNR we can have the following approximations

$$
\begin{aligned}
& \prod_{i=1}^{n}\left(1+\lambda_{M_{i}}\right) \simeq 1+\prod_{i=1}^{n} \lambda_{M_{i}} \\
& =1+\left(\frac{\delta_{s, r}^{2} P_{1} P_{2} / K_{n}}{4 N_{0} \quad P_{1} \delta_{s, r}^{2}+N_{o}+\frac{P_{2}}{K_{n}} \sum_{i=1}^{n}\left|h_{r_{i}, d}\right|^{2}}\right)^{n} \prod_{i=1}^{n} \lambda_{i}\left|h_{r_{i}, d}\right|^{2} \\
& \simeq \prod_{i=1}^{n}\left(1+\frac{\delta_{s, r}^{2} P_{1} P_{2} / K_{n}}{4 N_{0} \quad P_{1} \delta_{s, r}^{2}+N_{o}+\frac{P_{2}}{K_{n}} \sum_{i=1}^{n}\left|h_{r_{i}, d}\right|^{2}} \lambda_{i}\left|h_{r_{i}, d}\right|^{2}\right),
\end{aligned}
$$

where $\lambda_{i}$ are the eigenvalues of the $\Psi(\mathbf{X}, \hat{\mathbf{X}})$ matrix. We have used the fact that the determinant of a matrix equals the product of the matrix eigenvalues and that the determinant of the multiplication of two matrices equals the product of the individual matrices' determinants.

The PEP in (23) can now be approximated at high SNR as

$$
\begin{aligned}
& P(\mathbf{X} \rightarrow \hat{\mathbf{X}}) \leq E_{h_{r_{1}, d}, \cdots, h_{r_{n}, d}}[ \\
& \left.\frac{1}{\prod_{i=1}^{n}\left(1+\frac{\delta_{s, r}^{2} P_{1} P_{2} / K_{n}}{4 N_{0}\left(P_{1} \delta_{s, r}^{2}+N_{o}+\frac{P_{2}}{K_{n}} \sum_{i=1}^{n}\left|h_{r_{i}, d}\right|^{2}\right)} \lambda_{i}\left|h_{r_{i}, d}\right|^{2}\right)}\right] .
\end{aligned}
$$

Consider now the term $h=\sum_{i=1}^{n}\left|h_{r_{i}, d}\right|^{2}$ in (25) which can be reasonably approximated as $\sum_{i=1}^{n}\left|h_{r_{i}, d}\right|^{2} \approx n \delta_{r, d}^{2}$, especially for large $n$ [5] (by the strong law of large numbers). Averaging the expression in (25) over the exponential distribution of $\left|h_{r_{i}, d}\right|^{2}$ we get

$$
\begin{aligned}
& P(\mathbf{X} \rightarrow \hat{\mathbf{X}}) \leq \prod_{i=1}^{n}\left(\frac{\left(\delta_{s, r}^{2} P_{1} P_{2} / K_{n}\right) \lambda_{i}}{4 N_{0}\left(P_{1} \delta_{s, r}^{2}+N_{o}+\frac{P_{2}}{K_{n}} \delta_{r, d}^{2} n\right)}\right)^{-1} \\
& \cdot \prod_{i=1}^{n}\left[-\exp \left(-\frac{4 N_{0}\left(P_{1} \delta_{s, r}^{2}+N_{o}+\frac{P_{2}}{K_{n}} \delta_{r, d}^{2} n\right)}{\left(\delta_{s, r}^{2} P_{1} P_{2} / K_{n}\right) \lambda_{i}}\right)\right. \\
& \left.\cdot \mathbf{E i}\left(-\frac{4 N_{0}\left(P_{1} \delta_{s, r}^{2}+N_{o}+\frac{P_{2}}{K_{n}} \delta_{r, d}^{2} n\right)}{\left(\delta_{s, r}^{2} P_{1} P_{2} / K_{n}\right) \lambda_{i}}\right)\right]
\end{aligned}
$$

where $\mathbf{E i}($.$) is the exponential integral function defined as$ [9]

$$
\mathbf{E i}(\mu)=\int_{-\infty}^{\mu} \frac{\exp (t)}{t} d t, \quad \mu<0
$$

The exponential integral function can be approximated as $\mu$ tends to 0 as $-\mathbf{E i}(\mu) \approx \ln \left(-\frac{1}{\mu}\right), \mu<0$ [9]. At high SNR (high $P$ ) we can get the bound in (26) as

$$
\begin{aligned}
P(\mathbf{X} \rightarrow \hat{\mathbf{X}}) \leq & \prod_{i=1}^{n}\left(\frac{\left(\delta_{s, r}^{2} P_{1} P_{2} / K_{n}\right) \lambda_{i}}{4 N_{0}\left(P_{1} \delta_{s, r}^{2}+\frac{P_{2}}{K_{n}} \delta_{r, d}^{2} n\right)}\right)^{-1} \\
& \cdot \prod_{i=1}^{n} \ln \left(\frac{\left(\delta_{s, r}^{2} P_{1} P_{2} / K_{n}\right) \lambda_{i}}{4 N_{0}\left(P_{1} \delta_{s, r}^{2}+\frac{P_{2}}{K_{n}} \delta_{r, d}^{2} n\right)}\right) .
\end{aligned}
$$

Let $P_{1}=\alpha P$ and $P_{2}=(1-\alpha) P$, where $P$ is the power per symbol, for some $\alpha \in(0,1)$. With the definition of the SNR as $S N R=P / N_{0}$, the bound in (28) can be given as

$$
P(\mathbf{X} \rightarrow \hat{\mathbf{X}}) \leq a_{A F} \frac{1}{\prod_{i=1}^{n} \lambda_{i}} S N R^{-n}(\ln (S N R))^{n},
$$


where $a_{A F}$ is a constant that depends on the power allocation parameter $\alpha$ and the channels' variances. The diversity order of the system can now be calculated as $d_{A F}=\lim _{S N R \rightarrow \infty}-\frac{\log (P E P)}{\log (S N R)}=n$. The system will achieve a full diversity of order $n$, if the matrix $\mathbf{M}$ is full rank, that is the code matrix $\Psi(\mathbf{X}, \hat{\mathbf{X}})$ must have a full rank of order $n$ over all distinct pairs of codewords $\mathbf{X}$ and $\hat{\mathbf{X}}$. It can be easily seen, following the same approach, that if the code matrix $\Psi(\mathbf{X}, \hat{\mathbf{X}})$ is rank deficient the system will not achieve full diversity. So any code that is designed to achieve full diversity in MIMO channels will achieve full diversity in the case of AAF distributed space-time coding. If the full diversity is achieved, the coding gain is $C_{A F}=\left(a_{A F} \frac{1}{\prod_{i=1}^{n} \lambda_{i}}\right)^{-\frac{1}{n}}$. To maximize the coding gain of the AAF distributed space-time codes we need to maximize the term $\prod_{i=1}^{n} \lambda_{i}$ which is the same as the determinant criterion used for MIMO channels [6].

\section{Simulation Results}

In this section, we present some simulations to verify the theoretical results presented in the previous sections. In the simulations, we take the variance of any source-relay or relay-destination channel to be 1 . We compare the performance of different systems with two relays helping the source. Fig. 1 shows the simulations for two DAF systems using the Alamouti scheme (DAF Alamouti) [7] and the diagonal STC (DAF DAST) [8], and the diagonal distributed space-time codes (DDSTC) with the AAF protocol. For fair comparison we fix the number of transmitted bits per symbol to be 1 bit/sym. Clearly the DAF system in general outperforms the AAF based system. Intuitively, the DAF will deliver signals that are less noisy to the destination because they suppress the noise by transmitting a noise free version of the signal. The AAF delivers more noise to the destination, due to noise propagation from the relay nodes, than the DAF system. The assumption of correct decision at the relay nodes (whether the relay has decoded correctly or not) imposes practical limitations on the DAF systems in general otherwise we may have error propagation [2] which will highly degrade the system SER performance.

\section{CONCLUSION}

In this paper, We derive the code design criteria for different systems based on PEP analysis. The different systems differ in the processing performed at the relay nodes. For the DAF distributed space-time codes we find that any code that is designed to achieve full diversity in the MIMO channels can achieve full diversity under the assumption that the relay nodes can decide whether they have decoded correctly or not. We prove that a code that maximizes the coding gain in the MIMO channels is not guaranteed to maximize the coding gain in the DAF

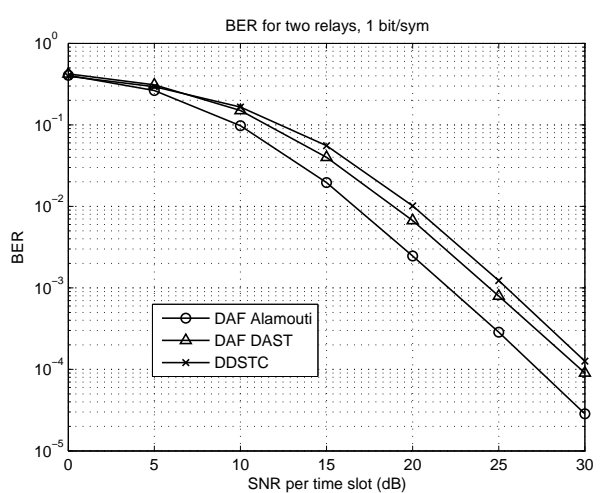

Fig. 1. BER for two relays with rate $1 \mathrm{bit} / \mathrm{sym}$.

distributed space-time coding due to the fact that not all the relays will always transmit their code columns in the second phase. Then, we consider the code design criteria for the AAF distributed space-time codes. In this case, the code designed to achieve full diversity in the MIMO channels will also achieve full diversity. Furthermore, the code that maximizes the coding gain in the MIMO channels will also maximize the coding gain in the AAF distributed space-time system.

\section{REFERENCES}

[1] J. N. Laneman and G. W. Wornell, "Distributed space-time coded protocols for exploiting cooperative diversity in wireless networks", IEEE Trans. Information Theory, vol. 49, pp. 24152425, Oct. 2003.

[2] J. N. Laneman, D. N. C. Tse and G. W. Wornell, "Cooperative diversity in wireless networks: Efficient protocols and outage behavior", IEEE Trans. Information Theory, vol. 50, no. 12, pp. 3062-3080, Dec. 2004.

[3] B. Sirkeci-Mergen and A. Scaglione, "Randomized distributed space-time coding for cooperative communication in selforganized networks," IEEE Workshop on Signal Processing Advances in Wireless Communications (SPAWC), New York, NY, June 5-8, 2005.

[4] S. Barbarossa, L. Pescosolido, D. Ludovici, L. Barbetta, and G. Scutari, "Cooperative wireless networks based on distributed space-time coding," in Proc. IEEE International Workshop on Wireless Ad-hoc Networks (IWWAN), Oulu, Finland, May 31-June 3, 2004.

[5] Y. Jing and B. Hassibi, "Distributed space-time coding in wireless relay networks", the 3rd IEEE Sensor Array and Multi-Channel Signal Processing Workshop, Sitges, Barcelona, Spain, July 18-21, 2004.

[6] V. Tarokh, N. Seshadri and A.R. Calderbank, "Space-time codes for high data rates wireless communications: Performance criterion and code construction," IEEE Trans. Information Theory, vol. 44, no. 2, pp. 744-765, Mar. 1998.

[7] S. Alamouti, "A simple transmit diversity technique for wireless communications", IEEE Journal on Select Areas in Communications, vol. 16, no. 8, pp. 1451-1458, Oct. 1998.

[8] M. O. Damen, K. Abed-Meraim, and J. C. Belfiore, "Diagonal algebraic space-time block codes", IEEE Trans. Information Theory, vol. 48, no. 2, pp. 628-636, Mar. 2002.

[9] I. S. Gradshteyn and I. M. Ryshik, Table of Integrals, Series and Products Academic Press, 6nd ed., 2000. 